Discussion Paper Series A No.549

\title{
Profits and Exploitation: A Reappraisal
}

\author{
Naoki Yoshihara \\ (Institute of Economic Research, Hitotsubashi University) \\ and \\ Roberto Veneziani \\ (Department of Economics, Queen Mary University of London)
}

April 2011

Institute of Economic Research

Hitotsubashi University

Kunitachi, Tokyo, 186-8603 Japan 


\title{
Profits and Exploitation: A Reappraisal ${ }^{*}$
}

\author{
Naoki Yoshihara $^{\dagger} \quad$ Roberto Veneziani ${ }^{\ddagger}$
}

April 2011

\begin{abstract}
This paper provides a mathematical analysis of the Marxian theory of the exploitation of labour in general equilibrium models. The two main definitions of Marxian exploitation in the literature, proposed by Morishima (1974) and Roemer (1982), respectively, are analysed in the context of general convex economies. It is shown that, contrary to the received view, in general these definitions do not preserve the so-called Fundamental Marxian Theorem (FMT), which states that the exploitation of labour is synonymous with positive profits. A new definition of Marxian labor exploitation is proposed, which is shown to preserve the FMT in general convex economies, in equilibrium.
\end{abstract}

JEL Classification Numbers: D31, D46, B51, B24.

Keywords: exploitation, profits, reproducible solutions.

\section{Introduction}

The derivation of a general relation between the exploitation of labour and the existence of profits, and the definition of an appropriate notion of general equilibrium have historically been central (and partly related) issues in

\footnotetext{
*We would like to thank John Roemer and two anonymous referees for useful comments and suggestions on an earlier draft. The usual disclaimer applies.

$\dagger$ (Corresponding author) The Institute of Economic Research, Hitotsubashi University, Naka 2-1, Kunitachi, Tokyo 186-0004, Japan. Phone: (81)-42-580-8354, Fax: (81)-42-5808333. e-mail: yosihara@ier.hit-u.ac.jp

$\ddagger$ Department of Economics, Queen Mary University of London, Mile End Road, London E1 4NS, United Kingdom. (r.veneziani@qmul.ac.uk)
} 
mathematical Marxian economics. From a theoretical viewpoint, this is due to the fundamental relevance of the theory of exploitation, and of the notion of the reproducibility of an economic system in Marxian approaches. From a formal viewpoint, this is explained by the difficulty of proving some core propositions in exploitation theory which Marx himself, and the early Marxists, took for evident.

To be precise, a key tenet of Marxist theory is the so-called Fundamental Marxian Theorem (hereafter, FMT) which establishes a correspondence between a positive profit rate and the existence of exploitation. This theorem was originally proved by Okishio (1963), and later named as such by Morishima (1973), in the context of simple Leontief economies with homogeneous labour. Yet, outside of stylised, linear two-class economies, both the appropriate definition of exploitation and the validity of the core insights of exploitation theory, including the FMT, are not uncontroversial, and indeed a number of approaches have been proposed in an attempt to generalise the FMT (see Yoshihara, 2010).

This has generated a substantial literature. Whereas the FMT was successfully extended to Leontief economies with heterogeneous labour by Fujimori (1982), Krause (1982), and others, in a famous book Steedman (1977) proved that the FMT does not hold in more general von Neumann economies. One solution was proposed by Morishima (1974) based on a new definition of exploitation, and focusing on von Neumann's notion of Balanced Growth Equilibrium. Yet Roemer (1981) showed that if Morishima's (1974) definition is adopted, the FMT does not hold, in general, in economies with a convex cone technology, if a different notion of equilibrium is adopted, namely that of reproducible solution (Roemer, 1980), unless some restrictions on the production set are imposed. Later, Roemer (1982) proposed an alternative definition of exploitation, which according to him would generalise the FMT (and other key Marxian propositions) to convex cone economies at reproducible solutions.

Various authors have objected to Morishima's (1974) and Roemer's (1982) definitions of exploitation on theoretical and exegetical grounds and other approaches have been proposed (for recent debates, see Veneziani, 2004; Flaschel 2010). Yet they remain the most prominent definitions in the literature, and this is to a significant extent due precisely to the formal derivation of the FMT. The relevance of the FMT, in fact, is such that although it is proved as a result, its epistemological status is that of a postulate: the appropriate definition of exploitation is widely considered to be one which preserves 
FMT. ${ }^{1}$ There are various reasons why the FMT is considered a key tenet of exploitation theory, and of Marxian economics in general. In the standard approach, the FMT is often interpreted as the formalisation of Marx's claim that profits are uniquely determined by exploitation in class divided economies. According to Roemer (1980, 1981), instead, the FMT captures the productiveness of capitalist economies and it provides the necessary and sufficient conditions for the existence of nontrivial general equilibria. From a normative perspective, however, the FMT is relevant because it captures a key insight of the theory of exploitation as the unequal exchange of labour, according to which exploitative relations are characterised by a difference between the amount of labour that an individual provides and the amount of labour contained in some relevant bundle that she does (or can) purchase with her income. From this perspective, exploitation theory focuses on inequalities in the distribution of income and labour, and thus it captures some core inequalities of well-being freedom (see Rawls, 1971; Sen, 1985), which are characteristic of capitalist economies. According to the FMT, profits play a key role in the generation of exploitation as the unequal exchange of labour (and thus of inequalities in well-being freedom), since they represent the way in which capitalists appropriate social surplus and social labour.

In this paper, the FMT is analysed in the context of general economies with convex cone production technologies, both assuming a representative agent and allowing for heterogeneous preferences over consumption. Within this general framework, the classic definitions of exploitation proposed by Morishima (1974) and Roemer (1982) are reconsidered, and it is shown that, contrary to the received wisdom, neither of them preserves the FMT in general. If the equilibrium notion is that of a reproducible solution, then the FMT does not hold under Roemer's definition, even if workers are identical and consume a uniform subsistence bundle. Further, if one allows for heterogeneity in workers' preferences, then the FMT does not hold under Morishima's definition, either. A new definition of exploitation is proposed which focuses on workers' income, rather than on their consumption bundle. This definition seems preferable on theoretical grounds, because the exploitation status of an agent is identified based on the objective features of an economy (including data on production, income, labour supply, etc.), rather than on the subjective and idiosyncratic factors driving consumption

\footnotetext{
${ }^{1}$ According to Roemer (1982), the Class-Exploitation Correspondence Principle plays a similar epistemological role. This is briefly discussed in the concluding section below.
} 
choices. As argued by Yoshihara and Veneziani (2011), Marxian exploitation theory is inherently objectivist in nature, and two agents with the same endowments, labour supply, and income should have the same exploitation status, regardless of their choice of a consumption bundle. Furthermore, it is shown that if this definition is adopted, the FMT holds in general convex economies both with a representative agent and if heterogeneous preferences are allowed for.

This paper is related to a small but growing recent literature on exploitation theory. A conceptually related definition, for example, has been proposed, and axiomatically derived, by Yoshihara (2010) and Yoshihara and Veneziani $(2009,2010)$. Moreover, although this paper focuses on static perfectly competitive economies, the mathematical Marxian exploitation theory has been recently extended to models of contested exchange (Yoshihara, 1998) and to dynamic economies with intertemporally optimising agents (Veneziani, 2007).

The rest of the paper is organised as follows. Section 2 outlines the basic model. Section 3 introduces the two main received definitions of Marxian exploitation and proves that the FMT does not hold in general under either of them. Section 4 presents a new definition of exploitation and shows that it preserves the FMT in general. Section 5 concludes.

\section{The Basic Model}

An economy consists of a set $H$ of agents, or households, who trade $m$ commodities. Let $\mathbb{R}$ be the set of real numbers, and let $\mathbb{R}_{+}$(resp., $\mathbb{R}_{++}$) be the set of nonnegative (resp., strictly positive) real numbers. Production technology is freely available to all agents, who can operate any activity in the production set $P$, which has elements of the form $\alpha=\left(-\alpha_{0},-\underline{\alpha}, \bar{\alpha}\right)$, where $\alpha_{0} \in \mathbb{R}_{+}$is the direct labour input; $\underline{\alpha} \in \mathbb{R}_{+}^{m}$ are the inputs of the produced goods; and $\bar{\alpha} \in \mathbb{R}_{+}^{m}$ are the outputs of the $m$ goods. Thus, elements of $P$ are vectors in $\mathbb{R}^{2 m+1}$. The net output vector arising from $\alpha$ is denoted as $\widehat{\alpha} \equiv \bar{\alpha}-\underline{\alpha}$. Let the vector with all components equal to zero be denoted as 0. The following assumptions on $P$ hold throughout the paper. ${ }^{2}$

\footnotetext{
${ }^{2}$ For all vectors $x, y \in \mathbb{R}^{p}, x \geqq y$ if and only if $x_{i} \geqq y_{i}(i=1, \ldots, p) ; x \geq y$ if and only if $x \geqq y$ and $x \neq y ; x>y$ if and only if $x_{i}>y_{i}(i=1, \ldots, p)$. Note that vectors are columns unless otherwise specified.
} 
Assumption 0 (A0). $P$ is a closed convex cone with vertex 0.

Assumption 1 (A1). For all $\alpha \in P$, if $\bar{\alpha} \geq 0$ then $\alpha_{0}>0$.

Assumption 2 (A2). For all $c \in \mathbb{R}_{+}^{m}$, there is an $\alpha \in P$ such that $\widehat{\alpha} \geqq c$.

A1 implies that labour is indispensable to produce any non-negative and non-zero output vector. A2 states that any non-negative commodity vector is producible as net output. It is worth stressing that $\mathrm{A} 0 \sim \mathrm{A} 2$ represent a significant generalisation of the standard linear technologies analysed in the literature on the FMT, which represent special cases of the model considered in this paper. For example, a von Neumann technology is defined by a tuple $(B, A, L)$ where $B$ is an $m \times n$ output matrix; $A$ is an $m \times n$ input matrix; and $L$ is a $1 \times n$ vector of labour coefficients, and the production possibility set $P_{(A, B, L)}$ is given by

$$
P_{(A, B, L)} \equiv\left\{\alpha \in \mathbb{R}^{2 m+1} \mid \exists x \in \mathbb{R}_{+}^{n}: \alpha \leqq(-L x,-A x, B x)\right\} .
$$

$P_{(A, B, L)}$ is a closed convex cone in $\mathbb{R}^{2 m+1}$ with $\mathbf{0} \in P_{(A, B, L)}$ and it satisfies $\mathrm{A} 1 \sim \mathrm{A} 2$.

Although different assumptions concerning agents' behaviour will be considered below, assumption A0 A2 will be retained throughout the paper. For the sake of simplicity, in what follows, economies whose production set satisfies $\mathrm{A} 0 \sim \mathrm{A} 2$ will be referred to as general convex economies.

Given $P$, the set of production activities feasible with $\alpha_{0}=k$ units of labour can be defined as follows:

$$
P\left(\alpha_{0}=k\right) \equiv\left\{\left(-\alpha_{0},-\underline{\alpha}, \bar{\alpha}\right) \in P \mid \alpha_{0}=k\right\},
$$

and the set of net output vectors feasible with $k$ units of labour is:

$$
\widehat{P}\left(\alpha_{0}=k\right) \equiv\left\{\widehat{\alpha} \in \mathbb{R}^{m} \mid \exists \alpha^{\prime} \in P\left(\alpha_{0}=k\right): \bar{\alpha}^{\prime}-\underline{\alpha}^{\prime} \geqq \widehat{\alpha}\right\} .
$$

For any set $X \subseteq \mathbb{R} \times \ldots \times \mathbb{R}, \partial X \equiv\left\{x \in X \mid \nexists x^{\prime} \in X\right.$ s.t. $\left.x^{\prime}>x\right\}$ is the frontier of $X$, and $X^{\circ} \equiv X \backslash \partial X$ is the interior of $X$.

Given a market economy, a (row) vector $p \in \mathbb{R}_{+}^{m}$ describes the price of each of the $m$ commodities in the economy. The nominal wage rate is normalised to one. For any agent $\nu \in H$, let $\omega^{\nu} \in \mathbb{R}_{+}^{m}$ denote her initial endowments. In the literature on the FMT, it is assumed that the set of agents $H$ can be partitioned into two disjoint subsets, namely the working class, denoted as $W$, which comprises agents with no initial endowments; and 
the set $N$ of capitalists, who own at least some productive assets. Formally, $W=\left\{\nu \in H \mid \omega^{\nu}=\mathbf{0}\right\}$ and $N=\left\{\nu \in H \mid \omega^{\nu} \geq \mathbf{0}\right\}$. Further, it is assumed that workers are endowed with one unit of (homogeneous) labour.

For a given price vector $p$ and wage rate $w=1$, capitalists are assumed to maximise profits subject to their wealth constraint. Formally, each $\nu \in N$ solves: ${ }^{3}$

$$
\begin{aligned}
\text { choose } \alpha^{\nu} & \in P \text { to maximise } p \bar{\alpha}^{\nu}-\left(p \underline{\alpha}^{\nu}+\alpha_{0}^{\nu}\right) \\
\text { s.t. } p \underline{\alpha}^{\nu}+\alpha_{0}^{\nu} & \leqq p \omega^{\nu} .
\end{aligned}
$$

The set of production processes that solve this problem is denoted by $\mathcal{A}^{\nu}(p, 1)$. In line with standard classical political economy, it is assumed that capitalists do not work and do not consume: they use their revenues to accumulate for production in the next period.

Following the standard literature on the FMT, workers are assumed to supply a fixed amount of labour, equal to their labour endowment, and to be abundant relative to social productive assets. This assumption reflects the Marxian view that involuntary unemployment is a structural feature of capitalist economies. Workers' consumption behaviour, instead, will be specified below, where two different models are considered, by assuming first that workers consume a fixed subsistence bundle, and then relaxing this assumption by endowing them with heterogeneous demand functions for commodities.

\section{The classic approaches to the FMT}

\subsection{Two definitions of exploitation}

In Marxian theory, exploitation is conceived of as the unequal exchange of labour between agents: considering a worker $\mu \in W$, exploitative relations are characterised by systematic differences between the labour contributed by $\mu$ to the economy and the labour 'received' by $\mu$, which is given by the amount of labour contained, or embodied, in some relevant consumption bundle(s).

\footnotetext{
${ }^{3}$ Thus, noting that inputs are traded at the beginning of the period and outputs at the end, the optimisation programme can be interpreted as incorporating an assumption of stationary expectations on prices (see Roemer 1980; 1981, Chapter 2).
} 
Therefore, for any bundle $c \in \mathbb{R}_{+}^{m}$, it is necessary to define the labour value (or labour content) of $c$. Outside of standard Leontief economies, the definition of the labour content of $c$ is not obvious, and various definitions have, in fact, been proposed. In this section, the two most prominent definitions in the literature are presented, namely the definitions proposed by Morishima (1974; see also Roemer, 1981), and Roemer (1982).

In Morishima's (1974) approach, the labour value of a bundle of goods is independent of the equilibrium the economy is in. For any $c \in \mathbb{R}_{+}^{m}$, let

$$
\phi(c) \equiv\{\alpha \in P \mid \widehat{\alpha} \geqq c\},
$$

denote the set of activities that produce at least $c$ as net output. Then:

Definition 1 (Morishima, 1974): The labour value of a bundle c, l.v. (c), is

$$
\text { l.v. }(c) \equiv \min \left\{\alpha_{0} \mid \alpha=\left(-\alpha_{0},-\underline{\alpha}, \bar{\alpha}\right) \in \phi(c)\right\} \text {. }
$$

Therefore, if a worker spends one unit of labour and buys the bundle $c \in \mathbb{R}_{+}^{m} \backslash\{\mathbf{0}\}$, the rate of labour exploitation is defined as follows:

Definition 2 (Morishima, 1974): The rate of labour exploitation at a consumption bundle $c \in \mathbb{R}_{+}^{m} \backslash\{\mathbf{0}\}$ is

$$
e(c) \equiv \frac{1-l \cdot v \cdot(c)}{l . v \cdot(c)}
$$

It is easy to see that $\phi(c)$ is non-empty by A2, and that the set $\left\{\alpha_{0} \mid \alpha \in \phi(c)\right\}$ is bounded below by 0 , by the assumption $\mathbf{0} \in P$ and A1. Thus, l.v. (c) is well-defined since $P$ is closed. Moreover, by A1, l.v. $(c)$ is positive whenever $c \neq \mathbf{0}$, so that $e(c)$ is well-defined.

Morishima (1974) showed that, in the balanced growth equilibrium of a von Neumann linear economy with joint production, the warranted rate of profit is positive if and only if the rate of exploitation in Definition 2 is positive. This result holds even if the von Neumann technology contains inferior production processes. Roemer (1981) proved that if the more general equilibrium concept of reproducible solution (see Definition 5 below) is adopted, then the FMT holds under Definition 2 in economies with general convex technologies, provided inferior processes are ruled out. Roemer (1981) called the latter assumption Independence of Production (see Assumption 3 below). 
These results would seem to settle the issue of the validity of the FMT, and to provide strong support to Definition 2. According to Romer (1982), however, Definition 2 does not preserve other key axioms of Marxian exploitation theory (such as the correspondence between class and exploitation status) and this led him to propose an alternative definition. Unlike in Morishima (1974), the definition of labour value in Roemer (1982) depends on the specific equilibrium the economy is in. Given $(p, 1) \in \mathbb{R}_{+}^{m+1}$ and $\alpha \in P$, let $\pi(p, 1 ; \alpha) \equiv \frac{p \widehat{\alpha}-\alpha_{0}}{p \underline{\alpha}+\alpha_{0}}$ be the corresponding profit rate and let $\bar{P}(p, 1) \equiv \arg \max \{\pi(p, 1 ; \alpha) \mid \alpha \in P\}$ be the set of profit-rate-maximising production activities. Given $(p, 1) \in \mathbb{R}_{+}^{m+1}$ and a consumption bundle $c \in \mathbb{R}_{+}^{m}$ let

$$
\phi(c ; p, 1) \equiv\{\alpha \in \bar{P}(p, 1) \mid \widehat{\alpha} \geqq c\},
$$

denote the set of profit-rate-maximising activities which produce at least $c$ as net output. The labour value of a bundle $c$ is defined as follows:

Definition 3 (Roemer, 1982): The labour value of commodity vector $c$ at $(p, 1) \in \mathbb{R}_{+}^{m+1}$, l.v. $(c ; p, 1)$, is given by

$$
\text { l.v. }(c ; p, 1) \equiv \min \left\{\alpha_{0} \mid \alpha=\left(-\alpha_{0},-\underline{\alpha}, \bar{\alpha}\right) \in \phi(c ; p, 1)\right\} \text {. }
$$

The rate of labour exploitation is stated in the next definition.

Definition 4 (Roemer, 1982): The rate of labour exploitation at a consumption bundle $c \in \mathbb{R}_{+}^{m} \backslash\{\mathbf{0}\}$ and a price vector $(p, 1) \in \mathbb{R}_{+}^{m+1}$ is

$$
e(c ; p, 1) \equiv \frac{1-l . v \cdot(c ; p, 1)}{l . v .(c ; p, 1)}
$$

It is easy to verify that l.v. $(c ; p, 1)$ is well-defined if $\phi(c ; p, 1)$ is not empty, and it is positive whenever $c \neq \mathbf{0}$, so that $e(c ; p, 1)$ is well-defined. Also, l.v. $(c ; p, 1) \geqq$ l.v. $(c)$ so that $e(c ; p, 1)>0$ implies $e(c)>0 .{ }^{4}$

Although Roemer (1982) does not provide an explicit formal analysis of the FMT using Definition 4, he argues that Definition 4 is superior to the

\footnotetext{
${ }^{4}$ Note that $\phi(c ; p, 1)$ may be empty; for instance, if $\bar{P}(p, 1)$ consists of production activities whose corresponding net outputs are semi-positive vectors, then $\phi(c ; p, 1)$ is empty whenever $c>\mathbf{0}$, so that $l . v .(c ; p, 1)$ cannot be defined. This issue need not concern us here since the set l.v. $(c ; p, 1)$ is never empty whenever equilibrium price vectors are considered, as in the analysis below.
} 
alternatives (including Definition 2) also because the FMT continues to hold, provided a suitable restriction on the production set is imposed (Roemer, 1982, p.158). To this issue we turn next.

\subsection{Roemer's (1982) definition reconsidered}

In this subsection, the standard model for the FMT is analysed. In line with the literature, it is assumed that every agent in the working class consumes an exogenously given subsistence vector $b \in \mathbb{R}_{+}^{m}$, per unit of labour supplied. Thus, in this subsection a convex economy is given by technology $P$, agents $H=W \cup N$, subsistence bundle $b$, and productive endowments $\Omega=\left(\omega^{1}, \omega^{2}, \ldots, \omega^{|N|}\right)$, and is denoted as $E(P, H, b, \Omega)$. The definition of equilibrium for $E(P, H, b, \Omega)$ can then be provided:

Definition 5 (Roemer, 1981, Definition 2.5, p.41): A reproducible solution (RS) for the economy $E(P, H, b, \Omega)$ is a pair $\left((p, 1),\left(\alpha^{\nu}\right)_{\nu \in N}\right)$, where $p \in \mathbb{R}_{+}^{m}$, such that:

(a) $\forall \nu \in N, \alpha^{\nu} \in \mathcal{A}^{\nu}(p, 1)$ (profit maximisation);

(b) $\widehat{\alpha} \geqq \alpha_{0} b$ (reproducibility), where $\widehat{\alpha} \equiv \sum_{\nu \in N}\left(\bar{\alpha}^{\nu}-\underline{\alpha}^{\nu}\right)$ and $\alpha_{0} \equiv \sum_{\nu \in N} \alpha_{0}^{\nu} ;$

(c) $p b=1$ (subsistence wage); and

(d) $\underline{\alpha}+\alpha_{0} b \leqq \omega$ (social feasibility), where $\underline{\alpha} \equiv \sum_{\nu \in N} \underline{\alpha}^{\nu}$ and $\omega \equiv \sum_{\nu \in N} \omega^{\nu}$.

Part (a) is standard and needs no further comment. Part (b) states that net output in every sector should at least be sufficient for employed workers' total consumption. This amounts to requiring that social endowments do not decrease, because (b) is equivalent to $\omega-\left(\underline{\alpha}+\alpha_{0} b\right)+\bar{\alpha} \geqq \omega$, where the right hand side is the social stocks at the beginning of the period, and the left hand side is the stocks at the beginning of next period. Given that workers are abundant relative to productive assets, part (c) states that unemployment drives the equilibrium real wage rate down to the subsistence level. Finally, part (d) requires that intermediate inputs and workers' consumption can be anticipated from current stocks, since wages are assumed to be paid in advance.

The existence of a RS is guaranteed by the following proposition. 
Proposition 1 (Roemer 1980; 1981): Let $b \in \mathbb{R}_{++}^{m}$. Under A1 and A2, $a$ $\mathrm{RS}$ exists for the economy $E(P, H, b, \Omega)$.

Next, the assumption of independence of production is stated (see Roemer, 1981, p.47), which rules out inferior production processes from $P$ :

Assumption 3 (A3). (Independence of Production) If $\left(-\alpha_{0},-\underline{\alpha}, \bar{\alpha}\right) \in P$ and $\mathbf{0} \leqq c \leq \widehat{\alpha}$, then there exists $\left(-\alpha_{0}^{\prime},-\underline{\alpha}^{\prime}, \bar{\alpha}^{\prime}\right) \in P$ such that $\widehat{\alpha}^{\prime} \geqq c$ and $\alpha_{0}^{\prime}<\alpha_{0}$.

Under A3, it can be shown that the FMT holds when Definition 2 is adopted:

Proposition 2 (Roemer, 1981, Theorem 2.11): Let $b \in \mathbb{R}_{++}^{m}$. Under A1 A3, the following statements are equivalent:

(i) $e(b)>0$;

(ii) there exists a $R S$ yielding positive total profits;

(iii) all $R S$ 's yield positive total profits.

A first important point to note is that if Definition 4 is adopted instead, it is not obvious at all, even under A3, that the FMT continues to hold, because $e(b)>0$ does not necessarily imply $e(b ; p)>0 .{ }^{5}$ Indeed, Roemer (1982, Chapter 5, p.158, footnote 6 ) suggested that A3 be modified to hold on $\bar{P}(p, 1)$ in order to prove the FMT using Definition 4. Formally:

Assumption $3^{\prime}\left(\mathbf{A 3}^{\prime}\right)$. Let $(p, 1)$ be the price vector at a $\mathrm{RS}$. If $\left(-\alpha_{0},-\underline{\alpha}, \bar{\alpha}\right) \in$ $\bar{P}(p, 1)$ and $\mathbf{0} \leqq c \leq \widehat{\alpha}$, then there exists $\left(-\alpha_{0}^{\prime},-\underline{\alpha}^{\prime}, \bar{\alpha}^{\prime}\right) \in \bar{P}(p, 1)$ such that $\widehat{\alpha}^{\prime} \geqq c$ and $\alpha_{0}^{\prime}<\alpha_{0}$.

Unlike A3, however, A3' seems rather uncompelling. A3 requires the elimination of inferior activities from possible production sets: this is a reasonable technological restriction on the feasible set, which implies no significant loss of generality. A3' eliminates a very large class of production sets, including, for example, all those sets in which there are RS's with a unique profitmaximising path. One example is given in the proof of Theorem 1 below (see the economy described in Figure 1), but $\mathrm{A} 3^{\prime}$ also rules out the entire class of production sets whose corresponding net output sets, $\widehat{P}\left(\alpha_{0}=1\right)$, are

\footnotetext{
${ }^{5}$ Note that if $(p, 1)$ is a $\mathrm{RS}, e(b ; p)$ is well-defined regardless of the property of $\bar{P}(p, 1)$. This is because in any $\mathrm{RS}$, it is always true that $\phi(b ; p, 1)$ is non-empty by the fact that for the aggregate social production $\alpha=\sum_{\nu \in N} \alpha^{\nu}$ under the RS, $\alpha \in \bar{P}(p, 1)$ and $\widehat{\alpha} \geqq \alpha_{0} b$ hold.
} 
representable by strictly concave and continuously differentiable functions in which any RS has a unique profit-maximising path. But this seems a rather significant loss of generality and the rationale for this restriction is quite unclear. Moreover, it is not possible in general to check whether or not a given economy satisfies A3' before characterising the set of RS's of the economy.

This implies that the robustness of the FMT using Definition 4 should be checked under A3, without appealing to the ad hoc version suggested by Roemer (1982). The following theorem, however, shows that even if A3 is assumed, the FMT does not hold in general under Definition 4.

Theorem 1: Under $A 1 \sim A 3$, there exists an economy $E(P, H, b, \Omega)$ such that every $R S\left((p, 1),\left(\alpha^{\nu}\right)_{\nu \in N}\right)$ yields positive total profits but $e(b ; p, 1)=0$.

Proof. 1. (The economy) Let $m=2, W=\{\mu\}$, and $N=\{\nu\}$. Let $b=(1,1)$ and let $\omega=\omega^{\nu}=(2,1)$. Finally, define the following production points:

$$
\begin{aligned}
& \alpha^{1}=\left(-\alpha_{0}^{1},-\underline{\alpha}^{1}, \bar{\alpha}^{1}\right)=(-1,(-2,-1),(2,3)) ; \\
& \alpha^{2}=\left(-\alpha_{0}^{2},-\underline{\alpha}^{2}, \bar{\alpha}^{2}\right)=(-1,(-1,0),(3,1)) ; \text { and } \\
& \alpha^{3}=\left(-\alpha_{0}^{3},-\underline{\alpha}^{3}, \bar{\alpha}^{3}\right)=(-1,(-1,-1),(4,1)) .
\end{aligned}
$$

Then, let $P$ be a closed, convex cone subset of $\mathbb{R}^{5}$ such that

1) $\mathbf{0} \in P$; and

2) $\operatorname{co}\left\{\alpha^{1}, \alpha^{2}, \alpha^{3}\right\}=P\left(\alpha_{0}=1\right)$, where $\operatorname{co} X$ is the convex hull of a set $X$. $P$ satisfies all the assumptions on the production set, including A3.

2. (The equilibria) We will now characterise the set of RSs. Note that:

$$
\forall p \in \triangle^{2} \backslash\{(1,0)\}, p \omega<p\left(\underline{\alpha}^{\prime}+b\right)\left(\forall \underline{\alpha}^{\prime} \in \operatorname{co}\left\{\underline{\alpha}^{1}, \underline{\alpha}^{2}, \underline{\alpha}^{3}\right\} \backslash\left\{\underline{\alpha}^{2}\right\}\right),
$$

where $\triangle^{2}$ is the two-dimensional simplex. Note also that, by Definition 5(b), if $((p, 1), \alpha)$ is a RS, then $\widehat{\alpha} \geqq \alpha_{0} b$. Let $\alpha^{12} \equiv \frac{1}{2} \alpha^{1}+\frac{1}{2} \alpha^{2}$ : then $\operatorname{co}\left\{\alpha^{12}, \alpha^{2}\right\}=$ $\left\{\alpha^{\prime} \in \partial P\left(\alpha_{0}=1\right) \mid \widehat{\alpha}^{\prime} \geqq b\right\}$. Thus, by the convex cone property of $P$, if $((p, 1), \alpha)$ is a RS, there exist $t \in(0,1]$ and $\alpha^{\prime} \in \operatorname{co}\left\{\alpha^{12}, \alpha^{2}\right\}$ such that $\alpha=t \alpha^{\prime}$.

Let $\triangle^{2}\left(\alpha^{2}\right) \equiv\left\{p \in \triangle^{2} \mid \frac{1}{3} \leqq p_{1} \leqq \frac{1}{2}, \frac{2}{3} \geqq p_{2} \geqq \frac{1}{2}\right\}$. If $p \notin \triangle^{2}\left(\alpha^{2}\right)$, it is immediate to check that there is no $\alpha^{\prime} \in \operatorname{co}\left\{\alpha^{12}, \alpha^{2}\right\}$ such that for some appropriate $t \in(0,1], t \alpha^{\prime}$ constitutes a profit maximiser at that price. Hence $p \notin \triangle^{2}\left(\alpha^{2}\right)$ cannot hold at a RS. Therefore consider $p \in \triangle^{2}\left(\alpha^{2}\right)$. 
If $p=\left(\frac{1}{3}, \frac{2}{3}\right)$, then $p \widehat{\alpha}^{1}-\alpha_{0}^{1}=p \widehat{\alpha}^{2}-\alpha_{0}^{2}=p \widehat{\alpha}^{\prime}-\alpha_{0}^{\prime}>p \widehat{\alpha}^{\prime \prime}-\alpha_{0}^{\prime \prime}$ for any $\alpha^{\prime} \in \operatorname{co}\left\{\alpha^{1}, \alpha^{2}\right\}$ and any $\alpha^{\prime \prime} \in \operatorname{co}\left\{\alpha^{2}, \alpha^{3}\right\} \backslash\left\{\alpha^{2}\right\}$. However, because of the property $(*)$, the capital constraint in the profit maximisation problem implies that $\alpha^{2}$ is the unique profit maximiser at the price $p=\left(\frac{1}{3}, \frac{2}{3}\right)$.

If $p=\left(\frac{1}{2}, \frac{1}{2}\right)$, then $p \widehat{\alpha}^{2}-\alpha_{0}^{2}=p \widehat{\alpha}^{\prime \prime}-\alpha_{0}^{\prime \prime}>p \widehat{\alpha}^{\prime}-\alpha_{0}^{\prime}$ for any $\alpha^{\prime} \in$ co $\left\{\alpha^{1}, \alpha^{2}\right\} \backslash\left\{\alpha^{2}\right\}$ and any $\alpha^{\prime \prime} \in \operatorname{co}\left\{\alpha^{2}, \alpha^{3}\right\}$. Thus, by the same reasons as in the above paragraph, $\alpha^{2}$ is the unique profit maximiser at the price $p=\left(\frac{1}{2}, \frac{1}{2}\right)$ also.

If $p$ is such that $\frac{1}{3}<p_{1}<\frac{1}{2}, \frac{2}{3}>p_{2}>\frac{1}{2}$, then $\alpha^{2}$ is the unique profit maximiser at that price.

In sum, at any RS, $\alpha=\alpha^{2}$ must hold. Indeed, for any $p^{*} \in \triangle^{2}\left(\alpha^{2}\right)$, it is immediate to check that $\left(\left(p^{*}, 1\right), \alpha^{2}\right)$ constitutes a RS. Moreover, in this case, $\pi\left(p^{*}, 1 ; \alpha^{2}\right)>0$.

Insert Figure 1 around here.

3. (FMT) We can now check that at any $\mathrm{RS}\left(\left(p^{*}, 1\right), \alpha^{2}\right)$, where $p^{*} \in$ $\triangle^{2}\left(\alpha^{2}\right), e\left(b ; p^{*}, 1\right)=0$ holds, whereas profits are positive. This is because $\bar{P}\left(p^{*}, 1\right)=\left\{t \alpha^{2} \in P \mid t \in \mathbb{R}_{++}\right\}$and $\phi\left(\alpha_{0}^{2} b ; p^{*}, 1\right)=\left\{\alpha^{2}\right\}$, which implies l.v. $\left(\alpha_{0}^{2} b ; p^{*}, 1\right)=\alpha_{0}^{2}$, so that l.v. $\left(b ; p^{*}, 1\right)=1$.

Remark: Note that in the above proof of Theorem 1, though the constructed economy has $b \in \mathbb{R}_{++}^{m}$, this proof can be applied even to the case of $b=(0,1)$, so that the result does not depend on the assumption of $b \in \mathbb{R}_{++}^{m}$.

Theorem 1 proves that the FMT does not hold, in general, if Definition 4 is adopted, even under A3. It is actually worth noting that Theorem 1 does not only prove that there are economies in which at some equilibrium allocation the FMT does not hold. More strongly, and more significantly from a theoretical viewpoint, Theorem 1 shows that there are economies in which the FMT never holds in equilibrium if Roemer's (1982) definition is adopted. The intuition is the following: in the economy considered, at any $\operatorname{RS}\left((p, 1), \alpha^{2}\right)$, the activity $\alpha^{2}$ is the unique profit maximiser at $p$, and the corresponding net output $\widehat{\alpha}^{2}$ does not strictly dominate the subsistence bundle $b$, as described in Figure 1. In this case, the minimum amount of direct labour necessary to produce at least $b$ (among profit-maximising production activities) is equal to the amount of direct labour expended at the RS.

To be sure, the economy considered in the proof of Theorem 1 does not satisfy $\mathrm{A} 3^{\prime}$. But, as already noted, the theoretical relevance of $\mathrm{A} 3^{\prime}$ is dubious 
and indeed one may wonder whether the reason of the failure of the FMT may lie elsewhere. As argued in the Introduction, the specific notion of equilibrium adopted is quite important in the analysis of the FMT: for example, if A3 is dropped, Morishima's (1974) definition allows one to derive the FMT if one focuses on balanced growth equilibria, but not if RSs are considered. Then, one may argue that the definition of exploitation is correct, but the notion of equilibrium adopted, namely the RS, is inappropriate.

Arguably, Definition 5 captures various key aspects of the Marxian notion of reproducibility of an economy, including its emphasis on social feasibility (condition (d)). The latter aspect, however, represents an important departure from standard Walrasian notions of equilibrium and therefore it may be worth considering whether the FMT holds under Definition 4 by adopting an equilibrium notion without the explicit capital constraint. Formally, let: ${ }^{6}$

Definition 5*: A reproducible solution* (RS*) for the economy $E(P, H, b, \Omega)$ is a pair $\left((p, 1),\left(\alpha^{\nu}\right)_{\nu \in N}\right)$, where $p \in \mathbb{R}_{+}^{m}$, such that conditions (b) and (c) of Definition 5 hold, and:

$\left(\mathrm{a}^{*}\right) \forall \nu \in N, \alpha^{\nu} \in \mathcal{A}^{* \nu}(p, 1)$ (profit maximisation), where $\mathcal{A}^{* \nu}(p, 1) \equiv \arg \max \left\{p\left(\widehat{\alpha}^{\prime}-\alpha_{0}^{\prime} b\right) \mid \alpha^{\prime} \in P\right.$ and $\left.\alpha_{0}^{\prime} \leqq W^{\nu}\right\}$ and $W^{\nu}$ denotes $\nu$ 's financial endowment;

$\left(\mathrm{d}^{*}\right) \alpha_{0} \leqq \bar{L}$ (social feasibility of labour demand), where $\alpha_{0} \equiv \sum_{\nu \in N} \alpha_{0}^{\nu}$, and $\bar{L} \equiv \sum_{\nu \in N} W^{\nu}$.

The next Theorem provides partial support to the idea that the definition of equilibrium is relevant for establishing a correspondence between profits and exploitation.

Theorem 2: Let $b \in \mathbb{R}_{++}^{m}$. Under $A 1 \sim \mathrm{A} 3$, for any economy $E(P, H, b, \Omega)$, the following statements hold:

(i) there exists a $R S^{*}\left(\left(p^{*}, 1\right),\left(\alpha^{\nu}\right)_{\nu \in N}\right)$ such that $\left[p^{*}\left(\widehat{\alpha}^{*}-\alpha_{0}^{*} b\right)>0 \Rightarrow\right.$ $\left.e\left(b ; p^{*}, 1\right)>0\right]$

(ii) for any $R S^{*}\left((p, 1),\left(\alpha^{\nu}\right)_{\nu \in N}\right),\left[e(b ; p, 1)>0 \Rightarrow p\left(\widehat{\alpha}-\alpha_{0} b\right)>0\right]$.

Proof. 1. By Theorem 2.17 in Roemer (1981; Chapter 2; Appendix 2), we know that a $\mathrm{RS}^{*},\left((p, 1),\left(\alpha^{\nu}\right)_{\nu \in N}\right)$, exists.

\footnotetext{
${ }^{6}$ The equilibrium notion in Definition $5^{*}$ is based upon Roemer (1981, pp.65-67).
} 
2. At any $\mathrm{RS}^{*},\left((p, 1),\left(\alpha^{\nu}\right)_{\nu \in N}\right), \alpha_{0}=\bar{L}$ by profit maximisation, without loss of generality, and $p$ is an efficiency price which supports $\widehat{\alpha} \in \widehat{P}\left(\alpha_{0}=\bar{L}\right)$ as an efficient production point. In other words, $p \widehat{\alpha} \geqq p \widehat{\alpha}^{\prime}$ holds for any $\widehat{\alpha}^{\prime} \in \widehat{P}\left(\alpha_{0}=\bar{L}\right)$. Note that since $b \in \mathbb{R}_{++}^{m}$ and $\widehat{\alpha} \geqq \alpha_{0} b$, then $\widehat{\alpha} \in \mathbb{R}_{++}^{m}$. Hence, by A3, $p \in \mathbb{R}_{++}^{m}$ holds and therefore at a $\mathrm{RS}$

$$
p\left(\widehat{\alpha}-\alpha_{0} b\right)>0 \Leftrightarrow \widehat{\alpha}-\alpha_{0} b \geq \mathbf{0} .
$$

3. Show (ii). Take any RS*, $\left((p, 1),\left(\alpha^{\nu}\right)_{\nu \in N}\right)$, and suppose $p\left(\widehat{\alpha}-\alpha_{0} b\right)=$ 0 . Then, since $\widehat{\alpha}-\alpha_{0} b=\mathbf{0}$ and $\widehat{\alpha} \in \partial \widehat{P}\left(\alpha_{0}=\bar{L}\right)$, it follows that l.v. $\left(\alpha_{0} b\right)=$ $\bar{L}$. Hence, noting that l.v. $\left(\alpha_{0} b ; p, 1\right) \geqq$ l.v. $\left(\alpha_{0} b\right)$, it must be l.v. $\left(\alpha_{0} b ; p\right) \geqq \bar{L}$, so that $e(b ; p, 1) \leqq 0$.

4. Show (i). Let $b \in \partial \widehat{P}\left(\alpha_{0}=1\right)$. Then, it follows from A3 that, for any $\alpha \in P$ with $\widehat{\alpha}-\alpha_{0} b \geqq \mathbf{0}, \widehat{\alpha}-\alpha_{0} b=\mathbf{0}$ holds. Therefore, for any RS*, $\left((p, 1),\left(\alpha^{\nu}\right)_{\nu \in N}\right), p\left(\widehat{\alpha}-\alpha_{0} b\right)=0$.

Let $b \in \widehat{P}^{\circ}\left(\alpha_{0}=1\right)$. Then, there exists $\alpha^{*} \in \partial P$ such that $\widehat{\alpha}^{*}-\alpha_{0}^{*} b>$ 0. Let $p^{*} \in \mathbb{R}_{++}^{m}$ be a price vector which supports $\widehat{\alpha}^{*} \in \partial \widehat{P}\left(\alpha_{0}=\bar{L}\right)$ as an efficient production activity. Then, it is possible to construct a RS* $\left(\left(p^{*}, 1\right),\left(\alpha^{* \nu}\right)_{\nu \in N}\right)$ such that $\sum_{\nu \in N} \alpha^{* \nu}=\alpha^{*}$. This is because it is easy to find a division $\left(\alpha^{* \nu}\right)_{\nu \in N}$ of $\alpha^{*}$ such that $\alpha^{* \nu} \in \mathcal{A}^{* \nu}\left(p^{*}, 1\right)$ and $\sum_{\nu \in N} \alpha_{0}^{* \nu}=\bar{L}$, given that initial wealth for capitalists is only to finance the purchase of labour power. At this $\mathrm{RS}^{*}$, since $\widehat{\alpha}^{*}-\alpha_{0}^{*} b>\mathbf{0}, p^{*}\left(\widehat{\alpha}^{*}-\alpha_{0}^{*} b\right)>0$ holds. Moreover, by A3, there exists $\alpha^{* *} \in P$ such that $\widehat{\alpha}^{* *}-\alpha_{0}^{*} b \geqq \mathbf{0}$ and $\alpha_{0}^{* *}<\alpha_{0}^{*}$. In particular, since $\widehat{\alpha}^{*}-\alpha_{0}^{*} b>\mathbf{0}$, we can choose $\alpha^{* *}=t \alpha^{*}$ with $t \in(0,1)$ by the cone property of $P$. In this case, $\alpha^{* *} \in \bar{P}\left(p^{*}, 1\right)$ so that $l . v .\left(\alpha_{0}^{*} b ; p^{*}, 1\right)<$ $\alpha_{0}^{*}$. This implies $e\left(b ; p^{*}, 1\right)>0$, as desired.

Theorem 2 proves that for every convex cone economy, there always exists one equilibrium allocation (as defined in Definition $5^{*}$ ) such that the correspondence between profits and exploitation holds. This result would seem to establish the robustness of the FMT under Definition 4, without any ad hoc restrictions on A3, provided the appropriate equilibrium concept is adopted. This conclusion is unwarranted, though, as Theorem 2 proves a weak FMT. In fact, for every economy, the weakening of the capitalists' wealth constraints enlarges the set of equilibria and allows one to find one RS* $^{*}$ such that the desired relation between profits and exploitation holds. Yet this is not true in general for every equilibrium allocation, even if Defin- 
ition $5^{*}$ is adopted, since equilibria similar to the one described in the proof of Theorem 1 are not ruled out.

Indeed, and this is the second limit of Theorem 2, it is possible that even if workers supply a constant amount of labour and receive the same bundle of wage goods, they may be exploited or not depending on the equilibrium aggregate production point. For instance, given $\alpha_{0}>0$ and $\alpha_{0} b>\mathbf{0}$, if $\widehat{\alpha}-\alpha_{0} b>\mathbf{0}$ at a $\mathrm{RS}^{*}$, then they are exploited, while if $\widehat{\alpha}^{\prime}-\alpha_{0} b \geq \mathbf{0}$ at another RS*, then economic relations may be nonexploitative. The latter situation may occur, for instance, if the boundary $\partial \widehat{P}\left(\alpha_{0}=1\right)$ is representable by a strictly concave and continuously differentiable function, because $\bar{P}\left(p^{\prime}, 1\right)$ corresponds to the set of vectors such that $\left(t \widehat{\alpha}^{\prime}, t \alpha_{0}\right)$ where $t \in \mathbb{R}_{++}$. This ambiguity seems rather dubious, at least if attention is restricted to equilibrium allocations in which capitalists maximise profits, since the exploitation status of workers should depend only upon the objective features of the labour contract, such as $\alpha_{0}$ and $\alpha_{0} b .^{7}$ Instead, the previous example suggests that the actual choice of the aggregate (equilibrium) net output vector may influence the workers' exploitation status even if their labour conditions are unchanged.

\subsection{Morishima's (1974) definition and workers' het- erogenous consumption demands}

The previous analysis suggests that the definition of exploitation proposed by Roemer (1982) is not superior to Morishima's (1974), at least as far as the FMT is concerned. Indeed, it fares strictly worse: if A3 is imposed, the FMT holds in general at any RS of any convex economy $E(P, H, b, \Omega)$ under Definition 2, but not under Definition 4. The next question, then, concerns whether this result can be extended to more general economies. Although the technologies allowed for are very general (and standard in microeconomic theory), the assumptions on workers' behaviour seem restrictive, as they rule out both workers' choice of consumption bundles and heterogeneity in preferences. And both are among the important features of advanced economies that make the issue of exploitation a contentious one today.

\footnotetext{
${ }^{7}$ If disequilibrium allocations are also considered, then capitalists' choices - which may turn out to be suboptimal ex post - may also be relevant for the determination of exploitation status. For a thorough discussion and an axiomatic defence of objectivism in the Marxian theory of exploitation, see Yoshihara and Veneziani (2011).
} 
In this subsection, the validity of the FMT is analysed in economies with heterogeneous workers, who have potentially different consumption demands. In standard Leontief economies, the assumption of heterogeneity of preferences has no implications for the validity of the FMT: a positive rate of profit prevails at a $\mathrm{RS}$ if and only if the average rate of exploitation of all workers is positive, which in turn holds if and only if each and every worker is exploited (see Roemer, 1981). The following analysis will show, however, that this result no longer holds once general convex economies with heterogeneous consumption demands are considered.

Let $T$ be the nonempty, finite set of types of workers with heterogeneous consumption demands and let the generic element of $T$ be denoted as $\tau$. Let $F(\tau) \in[0,1]$ be the fraction of workers of type $\tau$. By definition, $\sum_{\tau \in T} F(\tau)=1$. Given $p \in \mathbb{R}_{+}^{m}$, the consumption demand of the $\tau$-type worker per unit of income is denoted as $d^{\tau}(p) \in \mathbb{R}_{+}^{m}$. For all $\tau \in T$, the demand function $d^{\tau}(\cdot)$ is assumed to be derived from a continuous, strictly monotonic, strictly quasi-concave, and homothetic utility function, and $p d^{\tau}(p)=1$ for any $p \in \mathbb{R}_{+}^{m}$ normalised to $\sum_{j=1}^{m} p_{j}=1$.

Further, for all $\tau \in T, \alpha_{0}^{\tau}$ is the amount of labour spent by $\tau$-type workers. Therefore given $p \in \mathbb{R}_{+}^{m}$ and an aggregate production plan $\alpha \in P$, and noting that workers are abundant relative to productive assets, aggregate labour demanded $\alpha_{0}$ is equal to aggregate labour expended: $\sum_{\tau \in T} \alpha_{0}^{\tau}=\alpha_{0}$. Then, the average consumption demand of employed workers is defined by:

$$
d\left(p ;\left(\alpha_{0}^{\tau}\right)_{\tau \in T}\right) \equiv \frac{\sum_{\tau \in T} \alpha_{0}^{\tau} d^{\tau}(p)}{\alpha_{0}}
$$

Note that $p \cdot d\left(p ;\left(\alpha_{0}^{\tau}\right)_{\tau \in T}\right)=1$ by definition.

An economy is now specified by a list $E_{T}=\left(P ; N ; \Omega ; T ;(F(\tau))_{\tau \in T} ;\left(d^{\tau}(\cdot)\right)_{\tau \in T}\right)$, and in what follows it will be denoted simply as $E_{T}$ for the sake of notational simplicity. The equilibrium notion for the economies $E_{T}$ with heterogeneous workers' demands is formalised as follows:

Definition 6: A reproducible solution ${ }^{T}\left(\mathrm{RS}^{T}\right)$ for the economy $E_{T}$ is a pair $\left((p, 1),\left(\alpha^{\nu}\right)_{\nu \in N},\left(\alpha_{0}^{\tau}\right)_{\tau \in T}\right)$, where $p \in \mathbb{R}_{+}^{m}$, such that:

(a) $\forall \nu \in N, \alpha^{\nu} \in \mathcal{A}^{\nu}(p, 1)$ (profit maximisation);

(b) $\widehat{\alpha} \geqq \alpha_{0} d\left(p ;\left(\alpha_{0}^{\tau}\right)_{\tau \in T}\right)$ (reproducibility), where $\widehat{\alpha} \equiv \sum_{\nu \in N}\left(\bar{\alpha}^{\nu}-\underline{\alpha}^{\nu}\right)$ and $\alpha_{0} \equiv \sum_{\nu \in N} \alpha_{0}^{\nu}=\sum_{\tau \in T} \alpha_{0}^{\tau} ;$

(c) $\forall \tau \in T, p d^{\tau}(p)=1$; and 
(d) $\underline{\alpha}+\alpha_{0} d\left(p ;\left(\alpha_{0}^{\tau}\right)_{\tau \in T}\right) \leqq \omega$ (social feasibility), where $\underline{\alpha} \equiv \sum_{\nu \in N} \underline{\alpha}^{\nu}$ and $\omega \equiv \sum_{\nu \in N} \omega^{\nu}$.

Definition 6 is similar to Definition 5, except that the aggregate consumption demands of employed workers are endogenous and possibly heterogeneous.

The next result proves the existence of a $\mathrm{RS}^{T}$ for these general economies.

Proposition 3: For all $\tau \in T$, let $d^{\tau}(\cdot)$ be a strictly monotone, continuous demand function whose range is within the consumption set $C \subseteq \mathbb{R}_{+}^{m}$. Under $A 1$ and A2, a $\mathrm{RS}^{T}$ exists for the economy $E_{T}$.

Proof. Straightforward modification of the proof of Corollary 2.8 in Roemer (1981, p.44).

The next Theorem establishes a preliminary result concerning the correspondence between the existence of positive profits and the exploitation of the average worker, under Definition 2.

Theorem 3: Under $A 1 \sim A 3$, in the economy $E_{T}$, let $d^{\tau}(\cdot)$ be derived from continuous, strictly monotonic, strictly quasi-concave, and homethetic utility function defined on the consumption set $C \subseteq \mathbb{R}_{+}^{m}$, which can be either unbounded, or sufficiently large but bounded. Let $\left((p, 1),\left(\alpha^{\nu}\right)_{\nu \in N},\left(\alpha_{0}^{\tau}\right)_{\tau \in T}\right)$ be a $\mathrm{RS}^{T}$ for the economy $E_{T}$. Then, total profits are positive if and only if $e\left(d\left(p ;\left(\alpha_{0}^{\tau}\right)_{\tau \in T}\right)\right)>0$.

Proof. $(\Rightarrow)$ : Let $\left((p, 1),\left(\alpha^{\nu}\right)_{\nu \in N},\left(\alpha_{0}^{\tau}\right)_{\tau \in T}\right)$ be a $\mathrm{RS}^{T}$ with positive total profits:

$$
p \widehat{\alpha}-\alpha_{0}=p \cdot\left(\widehat{\alpha}-\alpha_{0} d\left(p ;\left(\alpha_{0}^{\tau}\right)_{\tau \in T}\right)\right)>0 .
$$

Since $p \in \mathbb{R}_{+}^{m}$ and $\widehat{\alpha} \geqq \alpha_{0} d\left(p ;\left(\alpha_{0}^{\tau}\right)_{\tau \in T}\right)$ by Definition $6(\mathrm{~b})$, the last strict inequality implies $\widehat{\alpha} \geq \alpha_{0} d\left(p ;\left(\alpha_{0}^{\tau}\right)_{\tau \in T}\right)$. Thus, by A3, l.v. $\left(\alpha_{0} d\left(p ;\left(\alpha_{0}^{\tau}\right)_{\tau \in T}\right)\right)<$ $\alpha_{0}$. By the convex cone property of $P$, l.v. $\left(d\left(p ;\left(\alpha_{0}^{\tau}\right)_{\tau \in T}\right)\right)<1$, which implies $e\left(d\left(p ;\left(\alpha_{0}^{\tau}\right)_{\tau \in T}\right)\right)>0$.

$(\Leftarrow)$ : Since there is no $\mathrm{RS}^{T}$ with negative total profits, let $\left((p, 1),\left(\alpha^{\nu}\right)_{\nu \in N},\left(\alpha_{0}^{\tau}\right)_{\tau \in T}\right)$ be a $\operatorname{RS}^{T}$ with zero aggregate profits. Thus, $p \cdot\left(\widehat{\alpha}-\alpha_{0} d\left(p ;\left(\alpha_{0}^{\tau}\right)_{\tau \in T}\right)\right)=$ 0 . By Definition $6(\mathrm{~b}), \widehat{\alpha} \geqq \alpha_{0} d\left(p ;\left(\alpha_{0}^{\tau}\right)_{\tau \in T}\right)$. If for some commodity $j$, $\widehat{\alpha}_{j}-\alpha_{0} d_{j}\left(p ;\left(\alpha_{0}^{\tau}\right)_{\tau \in T}\right)>0$, then it follows that $p_{j}=0$. However, since every worker has strictly monotone preferences, $p_{j}=0$ implies $\widehat{\alpha}_{j}-\alpha_{0} d_{j}\left(p ;\left(\alpha_{0}^{\tau}\right)_{\tau \in T}\right)<$ 0 , a contradiction. Thus, $\widehat{\alpha}=\alpha_{0} d\left(p ;\left(\alpha_{0}^{\tau}\right)_{\tau \in T}\right)$. 
Suppose l.v. $\left(\alpha_{0} d\left(p ;\left(\alpha_{0}^{\tau}\right)_{\tau \in T}\right)\right)<\alpha_{0}$. Then, l.v. $(\widehat{\alpha})<\alpha_{0}$, which implies that there exists $\alpha^{\prime} \in P$ such that $\widehat{\alpha}^{\prime} \geqq \widehat{\alpha}$ and $\alpha_{0}^{\prime}<\alpha_{0}$. Because of the cone property of $P$, there is $\alpha^{\prime \prime} \in P$ such that $\widehat{\alpha}^{\prime \prime}=\frac{\alpha_{0}}{\alpha_{0}^{\prime}} \widehat{\alpha}^{\prime}$ and $\alpha_{0}^{\prime \prime}=\alpha_{0}$. If $\widehat{\alpha}>0$, then $\widehat{\alpha}^{\prime \prime}>\widehat{\alpha}$, so that $\frac{\widehat{\alpha}}{\alpha_{0}} \in \widehat{P}^{\circ}\left(\alpha_{0}=1\right)$ by $\frac{\widehat{\alpha}^{\prime \prime}}{\alpha_{0}} \in \partial \widehat{P}\left(\alpha_{0}=1\right)$. Let $\widehat{\alpha}_{i}^{\prime}=\widehat{\alpha}_{i}=0$ for some $i$. In this case, $\widehat{\alpha}^{\prime \prime}>\widehat{\alpha}$ does not hold. However, by A2, there exists $c \in \widehat{P}\left(\alpha_{0}=1\right) \cap \mathbb{R}_{++}^{m}$ which is sufficiently close to $\frac{\widehat{\alpha}^{\prime \prime}}{\alpha_{0}}$. Then, $c>\frac{\widehat{\alpha}}{\alpha_{0}}$ holds, so that $\frac{\widehat{\alpha}}{\alpha_{0}} \in \widehat{P}^{\circ}\left(\alpha_{0}=1\right)$. However, the last equation implies $d\left(p ;\left(\alpha_{0}^{\tau}\right)_{\tau \in T}\right) \in \widehat{P}^{\circ}\left(\alpha_{0}=1\right)$, since $\widehat{\alpha}=\alpha_{0} d\left(p ;\left(\alpha_{0}^{\tau}\right)_{\tau \in T}\right)$, thus $p\left[c-d\left(p ;\left(\alpha_{0}^{\tau}\right)_{\tau \in T}\right)\right]>0$ for some $c \in \widehat{P}\left(\alpha_{0}=1\right) \cap \mathbb{R}_{++}^{m}$ with $c>\frac{\widehat{\alpha}}{\alpha_{0}}$. This is a contradiction, since the $\operatorname{RS}^{T}\left((p, 1),\left(\alpha^{\nu}\right)_{\nu \in N},\left(\alpha_{0}^{\tau}\right)_{\tau \in T}\right)$ has zero aggregate profits. Thus, l.v. $\left(\alpha_{0} d\left(p ;\left(\alpha_{0}^{\tau}\right)_{\tau \in T}\right)\right)=\alpha_{0}$, so that $e\left(d\left(p ;\left(\alpha_{0}^{\tau}\right)_{\tau \in T}\right)\right)=0$.

Theorem 3 derives a general relation between profits and exploitation at the average, or aggregate, level. By Theorem 3, it is possible to conclude that the exploitation of the working class is a necessary and sufficient condition for the existence of exploitation. Yet, it is unclear that this result provides all the necessary information concerning the FMT. In fact, although workers here may have heterogeneous demand functions for consumption goods, they are identical in terms of their labour endowments and labour skills, their preferences for leisure, and other labour conditions. Therefore one may argue that a robust extension of the FMT should provide definite answers concerning the exploitation status of each and every worker. Although this issue is usually ignored in the literature, this is only due to the simplifying assumption of a representative worker. Arguably, the classic (albeit often implicit) understanding of the FMT is that it concerns all members of the working class, and not just the average worker.

So, the theoretically relevant question is whether, under Definition 2 , the FMT holds for each and every worker in general convex economies with heterogeneous consumption demands. The next result provides a necessary and sufficient condition for the FMT to hold in this sense.

Theorem 4: Under A1 A3, let $\left((p, 1),\left(\alpha^{\nu}\right)_{\nu \in N},\left(\alpha_{0}^{\tau}\right)_{\tau \in T}\right)$ be a $\mathrm{RS}^{T}$ for the economy $E_{T}$. Then, the following two statements are equivalent:

(i) total profits are positive if and only if $e\left(d^{\tau}(p)\right)>0$ for any $\tau \in T$;

(ii) total profits are positive if and only if $d^{\tau}(p) \in \widehat{P}^{\circ}\left(\alpha_{0}=1\right)$ for any $\tau \in T$. 
Proof. It suffices to prove that $d^{\tau}(p) \in \widehat{P}^{\circ}\left(\alpha_{0}=1\right)$ holds for any $\tau \in T$ if and only if $e\left(d^{\tau}(p)\right)>0$ for any $\tau \in T$. First, let $d^{\tau}(p) \in \widehat{P}^{\circ}\left(\alpha_{0}=1\right)$ hold for any $\tau \in T$. This implies for any $\tau \in T$, there exists $\widehat{\alpha} \in \partial \widehat{P}\left(\alpha_{0}=1\right)$ such that $\widehat{\alpha}>d^{\tau}(p)$. Then, since $P$ is a convex cone satisfying A2, there exists $\alpha^{*} \in P$ with $\alpha_{0}^{*}<1$, such that $\widehat{\alpha}^{*} \in \partial \widehat{P}\left(\alpha_{0}=\alpha_{0}^{*}\right)$ and $\widehat{\alpha}^{*} \geq d^{\tau}(p)$. This also implies $e\left(d^{\tau}(p)\right)>0$ for any $\tau \in T$.

Conversely, suppose that there exists $\tau^{\prime} \in T$ such that $d^{\tau^{\prime}}(p) \notin \widehat{P}^{\circ}\left(\alpha_{0}=1\right)$. Then, either $d^{\tau^{\prime}}(p) \in \partial \widehat{P}\left(\alpha_{0}=1\right)$ or $d^{\tau^{\prime}}(p) \notin P\left(\alpha_{0}=1\right)$. If $d^{\tau^{\prime}}(p) \notin$ $P\left(\alpha_{0}=1\right)$, then for any $\alpha^{\prime} \in P\left(\alpha_{0}=1\right), \widehat{\alpha}^{\prime} \not d^{\tau^{\prime}}(p)$ holds. If $d^{\tau^{\prime}}(p) \in$ $\partial \widehat{P}\left(\alpha_{0}=1\right)$, then it implies together with A3 that, for any $\alpha^{\prime} \in P\left(\alpha_{0}=1\right)$, $\widehat{\alpha}^{\prime} \nsucceq d^{\tau^{\prime}}(p)$. In sum, for any $\alpha^{\prime} \in P\left(\alpha_{0}=1\right)$, either $\widehat{\alpha}^{\prime}=d^{\tau^{\prime}}(p)$ or $\widehat{\alpha}^{\prime} \nsupseteq d^{\tau^{\prime}}(p)$ holds for this $\tau^{\prime} \in T$. This implies $e\left(d^{\tau^{\prime}}(p)\right) \leqq 0$ for this $\tau^{\prime} \in T$.

Theorem 4 states that, at any $\mathrm{RS}^{T}$, the correspondence between the existence of profits and the exploitation of every worker is equivalent to the correspondence between the existence of profits and the existence of some surplus labour - in the sense that each worker's optimal consumption bundle could be produced with strictly less labour than is actually supplied by the worker. Theorem 4 is interesting because it provides a general characterisation result, but also because it allows us to derive the main conclusion on Morishima's (1974) classic definition of exploitation. The next result, in fact, proves that if the latter definition is adopted, it is possible that profits are positive but some types of workers are not exploited. ${ }^{8}$

Corollary 1: Under $A 1 \sim A 3$, there exists an economy $E_{T}$ such that there exists a $\mathrm{RS}^{T},\left((p, 1),\left(\alpha^{\nu}\right)_{\nu \in N},\left(\alpha_{0}^{\tau}\right)_{\tau \in T}\right)$, which yields positive total profits but e $\left(d^{\tau^{*}}(p)\right)<0$ for some $\tau^{*} \in T$.

Proof. 1. (The economy) Let $m=2, N=\{\nu\}$, and $\omega=\omega^{\nu}=(2.5,0.75)$. Let $T=\left\{\tau, \tau^{\prime}\right\}$ with $F(\tau)=0.5=F\left(\tau^{\prime}\right)$, and let the demand functions $d^{\tau}(\cdot)$ and $d^{\tau^{\prime}}(\cdot)$ be derived from continuous, monotonic, quasi-concave, and

\footnotetext{
${ }^{8}$ In this paper, a negative exploitation rate simply implies that (some types of) workers are not exploited. In a more general model, however, one might define exploiters as those agents whose labour supplied is smaller than the value of their labour power. Based on such definition, Corollary 1 could be interpreted as suggesting that some propertyless workers are actually exploiters.
} 
homothetic utility functions. Furthermore let:

$$
d^{\tau}(p)=(0.5,1.25) \text { and } d^{\tau^{\prime}}(p)=(2.5,0.25) \text { if } p=\left(\frac{1}{3}, \frac{2}{3}\right) .
$$

Next, define the following four production points:

$$
\begin{aligned}
& \alpha^{1}=\left(-\alpha_{0}^{1},-\underline{\alpha}^{1}, \bar{\alpha}^{1}\right)=(-1,(-1,0),(2.5,1)) ; \\
& \alpha^{2}=\left(-\alpha_{0}^{2},-\underline{\alpha}^{2}, \bar{\alpha}^{2}\right)=(-1,(0,-1),(2.5,1.5)) ; \\
& \alpha^{3}=\left(-\alpha_{0}^{3},-\underline{\alpha}^{3}, \bar{\alpha}^{3}\right)=(-1,(-1.5,0),(1.5,1.01)) ; \text { and } \\
& \alpha^{4}=\left(-\alpha_{0}^{4},-\underline{\alpha}^{4}, \bar{\alpha}^{4}\right)=(-1,(0,-1.5),(2.6,1.5)) .
\end{aligned}
$$

The production possibility set of this economy, $P$, is a closed, convex cone subset of $\mathbb{R}^{5}$ such that

1) $\mathbf{0} \in P$;

2) $P\left(\alpha_{0}=1\right)=\operatorname{co}\left\{\alpha^{1}, \alpha^{2}, \alpha^{3}, \alpha^{4}\right\}$.

$P$ satisfies all the assumptions on the production set, including A3.

2. (Equilibrium) Given the above economy, we show that $\left(\left(p^{*}, 1\right), \alpha^{*}\right)=$ $\left(\left(\left(\frac{1}{3}, \frac{2}{3}\right), 1\right), \alpha^{1}\right)$ with $\alpha_{0}^{\tau}=\alpha_{0}^{\tau^{\prime}}=0.5$ is a $\operatorname{RS}^{T}$. First, note that $p^{*}$ is an efficiency price for $\alpha^{*}$. Thus, since $p^{*} \underline{\alpha}^{*}+\alpha_{0}^{*}=p^{*} \omega^{\nu}<p^{*} \underline{\alpha}+\alpha_{0}$ for any $\alpha \in P\left(\alpha_{0}=1\right) \backslash\left\{\alpha^{1}\right\}$, it follows that $\alpha^{*} \in \mathcal{A}^{\nu}\left(p^{*}, 1\right)$, and Definition 6(a) holds. Next, since $d^{\tau}\left(p^{*}\right)=(0.5,1.25)$ and $d^{\tau^{\prime}}\left(p^{*}\right)=(2.5,0.25)$, Definition $6(\mathrm{c})$ holds, too. Moreover, since $\alpha_{0}^{\tau}=\alpha_{0}^{\tau^{\prime}}=0.5$ then $d\left(p^{*} ;\left(\alpha_{0}^{\tau}, \alpha_{0}^{\tau^{\prime}}\right)\right)=$ $(1.5,0.75)$, so that $\widehat{\alpha}^{*}=(1.5,1) \geq(1.5,0.75)=\alpha_{0}^{*} \cdot d\left(p^{*} ;\left(\alpha_{0}^{\tau}, \alpha_{0}^{\tau^{\prime}}\right)\right)$, so that Definition 6(b) holds. Finally, $\underline{\alpha}^{*}+\alpha_{0}^{*} \cdot d\left(p^{*} ;\left(\alpha_{0}^{\tau}, \alpha_{0}^{\tau^{\prime}}\right)\right)=(2.5,0.75)=\omega$, so that Definition 6(d) holds.

\section{Insert Figure 2 around here.}

3. (FMT) This $\mathrm{RS}^{T}$ yields positive profits: $p^{*} \widehat{\alpha}^{*}-\alpha_{0}^{*}=\frac{1}{6}>0,{ }^{9}$ but employed workers of type $\tau$ are not exploited according to Definition 2 , since $d^{\tau}\left(p^{*}\right)>\widehat{\alpha}^{3}$, and thus $d^{\tau}\left(p^{*}\right) \notin \widehat{P}^{\circ}\left(\alpha_{0}=1\right)$ which implies $e\left(d^{\tau}\left(p^{*}\right)\right) \leqq 0$,

${ }^{9}$ Also, $e\left(d\left(p^{*} ;\left(\alpha_{0}^{\tau}, \alpha_{0}^{\tau^{\prime}}\right)\right)\right)>0$, since l.v. $\left(d\left(p^{*} ;\left(\alpha_{0}^{\tau}, \alpha_{0}^{\tau^{\prime}}\right)\right)\right)<1$. The last inequality follows from l.v. $\left(\widehat{\alpha}^{*}\right)=1, \widehat{\alpha}^{*} \geq d\left(p^{*} ;\left(\alpha_{0}^{\tau}, \alpha_{0}^{\tau^{\prime}}\right)\right)$, and for any $\widehat{\alpha}^{\prime}=\lambda \widehat{\alpha}^{1}+(1-\lambda) \widehat{\alpha}^{2} \in$ $\widehat{P}\left(\alpha_{0}=1\right)$ with any $\lambda \in\left(0, \frac{1}{2}\right), \widehat{\alpha}^{\prime}>d\left(p^{*} ;\left(\alpha_{0}^{\tau}, \alpha_{0}^{\tau^{\prime}}\right)\right)$ holds. 
by Theorem 4 . To prove that the latter inequality is strict, it suffices to note that $d^{\tau}\left(p^{*}\right)>\widehat{\alpha}^{3}$, with l.v. $\left(\widehat{\alpha}^{3}\right)=1$, implies l.v. $\left(d^{\tau}\left(p^{*}\right)\right)>1$.

Theorem 4 and Corollary 1 imply that outside of simple Leontief economies, and even under A3, Morishima's (1974) classic definition of exploitation does not preserve one of the key tenets of the Marxian theory of labour exploitation, namely the correspondence between profit making by capitalists and the exploitation of propertyless workers. The FMT does not hold in the sense that within the set of propertyless agents earning the same income and working the same amount of time, there may be some workers who are not exploited even if profits are positive. It is worth stressing the importance of the stronger interpretation of the FMT adopted: in the economy constructed in the proof of Corollary 1, workers spend the same amount of labour time with the same labour skills, earn the same wage rate, and face the same budget constraint. They are completely identical, except for the actual bundle consumed. In this situation, one would expect workers to have exactly the same exploitation status. Instead, Corollary 1 shows that if Definition 2 is adopted, some types of workers are exploited while others paradoxically emerge as exploiters in equilibrium, due to differences in their subjective consumption demands, even though all types of workers face the same objective labour conditions (identical labour supply, income, skills, and so on).

Finally, although Theorem 4 and Corollary 1 focus on the notion of $\mathrm{RS}^{T}$ formalised in Definition 6, similar results could be derived by focusing on Balanced Growth Equilibria as in Morishima (1974). The negative conclusions on the ability of Morishima's (1974) famous definition of exploitation to capture the core intuitions of Marxian exploitation theory do not depend on the specific equilibrium notion adopted.

\section{A New Definition of Labour Exploitation}

Given the epistemological status of the FMT in exploitation theory, the negative results derived in the previous section suggest that another definition must be found that preserves the core insights of Marxian theory. This is the task of this section. The key intuition of the following analysis is that, despite all of their differences, the definitions proposed by Morishima (1974) and Roemer (1982) suffer from the same conceptual and formal problem. For both approaches define the value of labour power - that is, the amount of 
labour 'received' by workers - based on the bundle of goods consumed by workers, thus making purely subjective and idiosyncratic factors central in exploitation theory. To be sure, in the analysis of the economy, the essential heterogeneity of workers should be taken into account, rather than assumed away as in the standard literature. Yet the definition of the value of labour power should depend on income, or purchasing power, rather than on the choice of a specific consumption bundle. Again, the notion of exploitation should be such that two agents who earn the same income by supplying the same amount of homogeneous labour are identified as having the same exploitation status, regardless of their consumption choices.

Formally, the labour value of a bundle of commodities is still given by Definition 3 above. However, the definition of the value of labour power focuses on the income received by workers and exploitation is measured by the difference between the (one unit of) labour supplied by every worker and the minimal amount of direct labour socially necessary to provide the agent with her income (per unit of labour). For any $\mu \in W$, let:

$$
B(p, 1) \equiv\left\{f^{\mu} \in \mathbb{R}_{+}^{m} \mid p f^{\mu}=1\right\} .
$$

$B(p, 1)$ is the set of bundles that a worker can purchase, per unit of labour performed, using up all her income. Then:

Definition 7: The rate of labour exploitation for worker $\mu \in W$ at $(p, 1) \in$ $\mathbb{R}_{+}^{m+1}$ is

$$
e^{\mu}(p, 1) \equiv \frac{1-\min _{f^{\mu} \in B(p, 1)} l \cdot v \cdot\left(f^{\mu} ; p, 1\right)}{\min _{f^{\mu} \in B(p, 1)} l \cdot v \cdot\left(f^{\mu} ; p, 1\right)} .
$$

It is easy to verify that the value of labour power $-\min _{f^{\mu} \in B(p, 1)} l . v .\left(f^{\mu} ; p, 1\right)$ - is well-defined, since for any $p \in \mathbb{R}_{+}^{m} \backslash\{\boldsymbol{0}\}$, there exists $f^{\mu} \in B(p, 1)$ such that $\phi\left(f^{\mu} ; p, 1\right) \neq \varnothing$. Moreover, as already discussed, it has a positive value whenever $p \in \mathbb{R}_{+}^{m} \backslash\{\mathbf{0}\}$, so that $e^{\mu}(p, 1)$ is well-defined.

Thus, the value of labour power in Definition 7 focuses not on the worker's consumption vector, but rather on the income she earned at a RS. By Definition 7, worker $\mu$ is exploited at a given RS $\left((p, 1),\left(\alpha^{\nu}\right)_{\nu \in N}\right)$ if and only if the minimum amount of labour socially necessary to provide $\mu$ with her (unit) labour income $w=1$, is less than unity.

The next results show that a general, robust relation between positive profits and the exploitation of each and every worker can be derived under Definition 7 . Theorem 5 focuses on the economy with homogeneous workers: 
Theorem 5: Under A1 and A2, let $\left((p, 1),\left(\alpha^{\nu}\right)_{\nu \in N}\right)$ be a RS for an economy $E(P, H, b, \Omega)$. Then, total profits are positive if and only if $e^{\mu}(p, 1)>0$ for every worker $\mu \in W$.

Proof. $(\Rightarrow)$ : Let $\left((p, 1),\left(\alpha^{\nu}\right)_{\nu \in N}\right)$ be a RS with positive total profits: $p \widehat{\alpha}-\alpha_{0}=p \cdot\left(\widehat{\alpha}-\alpha_{0} b\right)>0$. Since $p \in \mathbb{R}_{+}^{m}$ and $\widehat{\alpha} \geqq \alpha_{0} b$ by Definition $5(\mathrm{~b})$, this implies $\widehat{\alpha} \geq \alpha_{0} b$. Let $f \in \mathbb{R}_{+}^{m}$ be such that $p f=p b$ and $\alpha_{0} f=t \widehat{\alpha}$ for some $0<t<1$. Note that such bundle exists, since $p \cdot\left(\widehat{\alpha}-\alpha_{0} b\right)=$ $p \cdot\left(\widehat{\alpha}-\alpha_{0} f\right)>0$. Since l.v. $(\widehat{\alpha} ; p, 1) \leqq \alpha_{0}$, it follows from $t \in(0,1)$ that l.v. $(t \widehat{\alpha} ; p, 1)=$ l.v. $\left(\alpha_{0} f ; p, 1\right)<\alpha_{0}$. By linearity, l.v. $(f ; p, 1)<1$, which implies $\min _{\widehat{f} \in B(p, 1)}$ l.v. $(\widehat{f} ; p, 1)<1$, so that $e^{\tau}(p, 1)>0$ for every $\tau \in T$.

$(\Leftarrow)$ : Since there is no RS with negative total profits, let $\left((p, 1),\left(\alpha^{\nu}\right)_{\nu \in N}\right)$ be a RS such that $p \cdot\left(\widehat{\alpha}-\alpha_{0} b\right)=0$. By Definition $5(\mathrm{~b}), \widehat{\alpha} \geqq \alpha_{0} b$. Therefore, if $p \in \mathbb{R}_{++}^{m}$, then $\widehat{\alpha}=\alpha_{0} b$. Let $f \in \mathbb{R}_{+}^{m}$ be such that $p f=p b$ and $\alpha_{0} f=t \widehat{\alpha}$ for some $0<t \leqq 1$. Then, $p \cdot\left(\widehat{\alpha}-\alpha_{0} f\right)=0$ and $\alpha_{0} f=t \widehat{\alpha}$ imply that $t=1$. Note that at this $\operatorname{RS}\left((p, 1),\left(\alpha^{\nu}\right)_{\nu \in N}\right)$, any profit-rate-maximising production points $\alpha^{\prime} \in \bar{P}(p, 1) \cap \partial P\left(\alpha_{0}=1\right)$ has the property that $p \widehat{\alpha}^{\prime}=1$ (by Definition $5(\mathrm{a})$ and the convex cone property of $P$ ). Thus, for any $\alpha^{\prime} \in$ $\bar{P}(p, 1) \cap \partial P\left(\alpha_{0}=1\right), p \widehat{\alpha}^{\prime}=\frac{p \widehat{\alpha}}{\alpha_{0}}=p b$. This implies that for any $f \in \mathbb{R}_{+}^{m}$ such that $p f=p b$, l.v. $(f ; p, 1) \geqq 1$ holds. Hence, $\min _{\widehat{f} \in B(p, 1)} l . v \cdot(\widehat{f} ; p, 1)=1$, so that $e^{\tau}(p, 1)=0$ for every $\tau \in T$.

If $p \in \mathbb{R}_{+}^{m}$, it may be the case that $\widehat{\alpha} \geq \alpha_{0} b$. However, as $p \cdot\left(\widehat{\alpha}-\alpha_{0} b\right)=0$ and $\alpha^{\nu} \in \mathcal{A}^{\nu}(p, 1)$ for all $\nu \in N, b \in \partial \widehat{P}\left(\alpha_{0}=1\right)$ holds. By the same argument as in the case with $p \in \mathbb{R}_{++}^{m}$, for any $f \in \mathbb{R}_{+}^{m}$ such that $p f=p b$, l.v. $(f ; p, 1) \geqq 1$ holds. Thus, $\min _{\widehat{f} \in B(p, 1)} l . v .(\widehat{f} ; p, 1)=1$, so that $e^{\tau}(p, 1)=$ 0 for every $\tau \in T$.

It is worth noting that if Definition 7 is adopted, neither $\mathrm{A} 3^{\prime}$ nor $\mathrm{A} 3$ are necessary to prove the FMT. This result is confirmed by the next Theorem, which establishes the robustness of the FMT also in economies with heterogeneous consumption demands.

Theorem 6: Under A1 and A2, let $\left((p, 1),\left(\alpha^{\nu}\right)_{\nu \in N},\left(\alpha_{0}^{\tau}\right)_{\tau \in T}\right)$ be a $\mathrm{RS}^{T}$ for $E_{T}$. Then, total profits are positive if and only if $e^{\tau}(p, 1)>0$ for every $\tau \in T$.

Proof. By Definition 6(c), at a $\operatorname{RS}^{T}$, for any $\tau \in T, p d^{\tau}(p)=p \cdot d\left(p ;\left(\alpha_{0}^{\tau}\right)_{\tau \in T}\right)=$ 
1. Thus, for any $\tau, \tau^{\prime} \in T, \min _{f^{\tau} \in B(p, 1)}$ l.v. $\left(f^{\tau} ; p, 1\right)=\min _{f^{\tau^{\prime}} \in B(p, 1)} l . v \cdot\left(f^{\tau^{\prime}} ; p, 1\right)$, so that $e^{\tau}(p, 1)=e^{\tau^{\prime}}(p, 1)$ for any $\tau, \tau^{\prime} \in T$. Let $\left((p, 1),\left(\alpha^{\nu}\right)_{\nu \in N}\right)$ be a $\operatorname{RS}^{T}$ with aggregate net output $\widehat{\alpha}$ and labour expended $\alpha_{0}$. Let $\widehat{\alpha}^{0} \equiv \frac{\widehat{\alpha}}{\alpha_{0}}$. Then, as shown in the proof of Theorem 5 , there exists some $t \in(0,1]$ such that $p t \widehat{\alpha}^{0}=$ 1. Moreover, for any $\tau \in T, \min _{f^{\tau} \in B(p, 1)} l . v .\left(f^{\tau} ; p, 1\right) \leqq l . v .\left(t \widehat{\alpha}^{0} ; p, 1\right)$.

$(\Rightarrow)$ : Let $\left((p, 1),\left(\alpha^{\nu}\right)_{\nu \in N},\left(\alpha_{0}^{\tau}\right)_{\tau \in T}\right)$ be a $\operatorname{RS}^{T}$ with positive total profits: $p \widehat{\alpha}-\alpha_{0}=p \cdot\left(\widehat{\alpha}-\alpha_{0} d\left(p ;\left(\alpha_{0}^{\tau}\right)_{\tau \in T}\right)\right)>0$. Thus, $p \widehat{\alpha}^{0}>1=p t \widehat{\alpha}^{0}$, and so $1>t$. The latter inequality implies l.v. $\left(t \widehat{\alpha}^{0} ; p, 1\right)<1$ by the cone property of $P$, since $\widehat{\alpha}^{0} \in \partial \widehat{P}\left(\alpha_{0}=1\right)$ by profit maximisation. Thus, $\min _{\widehat{f} \in B(p, 1)} l . v \cdot(\widehat{f} ; p, 1)<1$, so that $e^{\tau}(p, 1)>0$ for every $\tau \in T$.

$(\Leftarrow)$ : Let $\left((p, 1),\left(\alpha^{\nu}\right)_{\nu \in N}\right)$ be a $\operatorname{RS}^{T}$ such that $p \widehat{\alpha}-\alpha_{0}=p \cdot\left(\widehat{\alpha}-\alpha_{0} d\left(p ;\left(\alpha_{0}^{\tau}\right)_{\tau \in T}\right)\right)=$ 0 . Then, $p \widehat{\alpha}^{0}=1=p t \widehat{\alpha}^{0}$, and so $t=1$. Then, using the same argument as in the $(\Leftarrow)$ part of the proof of Theorem 5 , we can see that for any $f \in \mathbb{R}_{+}^{m}$ such that $p f=1$, l.v. $(f ; p, 1) \geqq 1$ and l.v. $\left(t \widehat{\alpha}^{0} ; p, 1\right)=1$ hold. Thus, $\min _{\widehat{f} \in B(p, 1)} l . v .(\widehat{f} ; p, 1)=1$, so that $e^{\tau}(p, 1)=0$ for every $\tau \in T$.

\section{Conclusions}

This paper analyses the mathematical Marxian theory of exploitation, focusing on the correspondence between positive profits and the existence of exploitation, and on the notion of reproducible solution. It is shown that, contrary to the received view, neither of the two main definitions of exploitation in the literature - proposed, respectively, by Morishima (1974) and Roemer (1982) - preserves the Fundamental Marxian Theorem in general convex economies. Given the central theoretical and epistemological role of the FMT in the Marxian theory of exploitation, this raises serious doubts on both approaches. The main shortcoming of the received definitions is their focus on the specific consumption bundle chosen by workers in the definition of the value of labour power (the amount of labour 'received' by workers in exchange for their own labour expenditure), which makes exploitation status dependent on purely subjective factors. From this perspective, the two main received definitions arguably fail to describe a fundamental feature of the Marxian theory, namely class struggle under historical materialism, since they make the exploitation concept dependent on workers' subjective views of the world: two workers with the same income, labour supply, and en- 
dowments may have different exploitation status based on their idiosyncratic choices as consumers. ${ }^{10}$ An alternative definition is proposed, which defines the value of labour power based on workers' income. Under this new definition a robust correspondence between positive profits and the exploitation of labour can be established in general convex economies with heterogeneous agents.

Two final remarks are worth making at this point about the robustness of our conclusions and the appeal of the proposed definition of exploitation. First, the economic models analysed in this paper are more general than those usually considered in the literature on Marxian exploitation. Yet they still contain a number of fairly strong simplifying assumptions, such as the neglect of consumption/leisure tradeoffs, workers' savings, capitalists' consumption, skills heterogeneity, and so on. One may legitimately wonder whether the FMT would still hold under the definition of exploitation proposed if the latter assumptions are violated. This topic is the object of ongoing work (Veneziani and Yoshihara, 2010), but preliminary results suggest that the main conclusions of this paper are robust. The theoretical and analytical emphasis on income earned, rather than actual consumption bundles, allows for a significant generalisation of standard insights.

Second, this paper focuses on the FMT as a key property of Marxian theory, but Roemer (1982) argued that an analogous epistemological role is played by the Class-Exploitation Correspondence Principle (CECP): any definition of exploitation should be such that agents in the upper classes emerge as exploiters and agents in the lower classes are exploited. Yoshihara (2010) has shown that neither Morishima's (1974) nor Roemer's (1982) definition preserves the CECP in general convex cone economies. In particular, an agent in the capitalist class may not be an exploiter if Morishima's (1974) definition is adopted; whereas an agent in the working class may not be exploited if Roemer's (1982) definition is adopted. Instead, the definition proposed in this paper does preserve the CECP in general (see Yoshihara, 2010), and thus it seems superior to standard approaches in this respect, too.

\footnotetext{
${ }^{10} \mathrm{We}$ are grateful to an anonymous referee for pointing out the dubious relevance of subjective factors in Marxian theory.
} 


\section{References}

Flaschel, P. (2010): Topics in Classical Micro- and Macroeconomics, Springer, New York.

Fujimori, Y. (1982): Modern Analysis of Value Theory, Springer-Verlag, Berlin.

Krause, U. (1982): "Heterogeneous Labour and the Fundamental Marxian Theorem," Review of Economic Studies 48, 173-178.

Morishima, M. (1973): Marx's Economics, Cambridge University Press, Cambridge.

Morishima, M. (1974): "Marx in the Light of Modern Economic Theory," Econometrica 42, pp. 611-632.

Okishio, N. (1963): "A Mathematical Note on Marxian Theorems," Weltwirtschaftsliches Archiv.

Rawls, J. (1971): A Theory of Justice, Harvard University Press, Cambridge.

Roemer, J. E. (1980): "A general equilibrium approach to Marxian economics," Econometrica 48, pp. 505-530.

Roemer, J. E. (1981): Analytical Foundations of Marxian Economic Theory, Cambridge University Press, Cambridge.

Roemer, J. E. (1982): A General Theory of Exploitation and Class, Harvard University Press, Cambridge.

Sen, A.K. (1985): Commodities and Capabilities. North-Holland, Amsterdam.

Steedman, I. (1977): Marx after Sraffa, New Left Books, London.

Veneziani, R. (2004): "The Temporal Single-system Interpretation of Marx's Economics: A Critical Evaluation," Metroeconomica 55, 96-114.

Veneziani, R. (2007): "Exploitation and Time," Journal of Economic Theory 132, 189-207.

Veneziani, R. and N. Yoshihara (2010): "Exploitation and Profits: A General Axiomatic Approach in Convex Economies with Heterogeneous Agents," IER Discussion Paper Series, No. A. 542, The Institute of Economic Research, Hitotsubashi University. 
Yoshihara, N. (1998): "Wealth, Exploitation, and Labor Discipline in the Contemporary Capitalist Economy," Metroeconomica 49, pp. 23-61.

Yoshihara, N. (2010): "Class and Exploitation in General Convex Cone Economies," Journal of Economic Behavior and Organization 75, 281-296.

Yoshihara, N. and R. Veneziani (2009): "Exploitation as the Unequal Exchange of Labour: An Axiomatic Approach," Working paper No.655, Queen Mary University of London.

Yoshihara, N. and R. Veneziani (2011): "Strong Subjectivism in the Marxian Theory of Exploitation: A Critique," Metroeconomica 62, pp. 53-68. 
Figure1 Proof of Theorem1

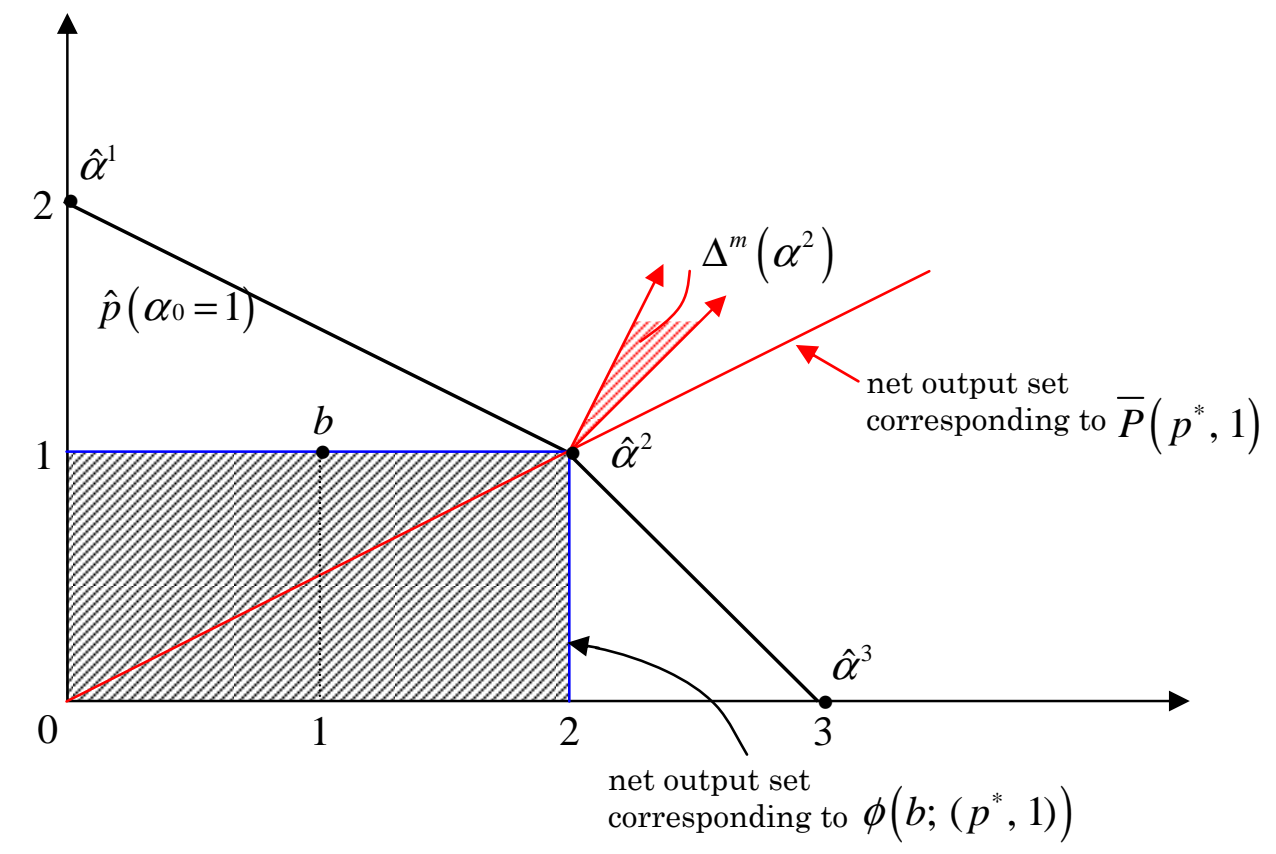


Figure2

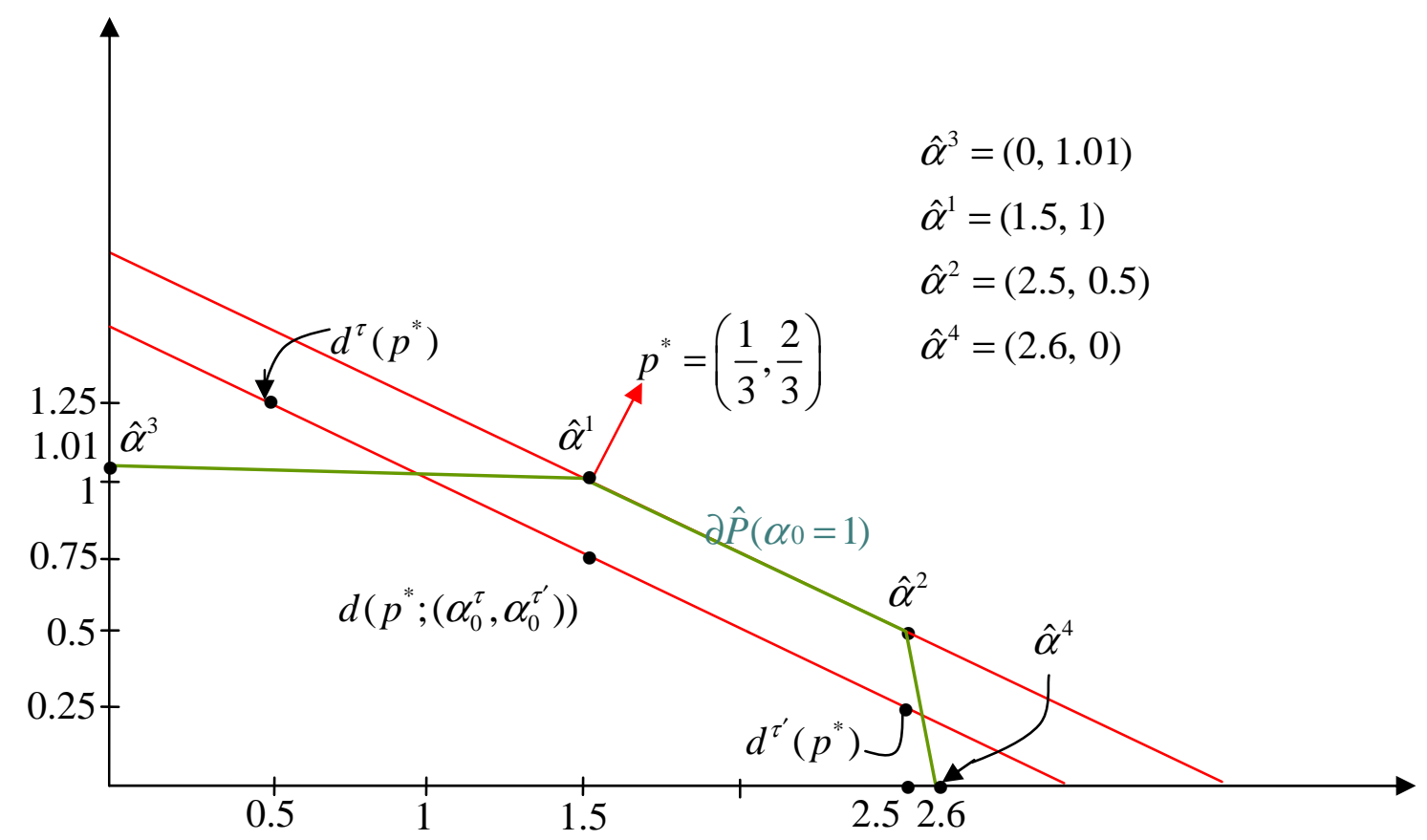

